\title{
Sensitive Impedimetric Immunoassay of Japanese Encephalitis Virus Based on Enzyme Biocatalyzed Precipitation on a Gold Nanoparticle-modified Screen-printed Carbon Electrode
}

\author{
Xiaohui Geng, ${ }^{*}$ Fanglin Zhang, ${ }^{* *}$ Qiang GAO, ${ }^{* \dagger}$ and Yingfeng LEI ${ }^{* * \dagger}$ \\ *Key Laboratory of Analytical Chemistry for Life Science of Shaanxi Province, School of Chemistry and \\ Chemical Engineering, Shaanxi Normal University, Xi'an, 710062, China \\ **Department of Microbiology, Faculty of Preclinical Medicine, The Fourth Military Medical University, \\ Xi'an, Shaanxi 710032, China
}

\begin{abstract}
A sensitive and disposable electrochemical impedance biosensor to detect Japanese encephalitis virus (JEV) was developed based on a gold nanoparticle (AuNP)-modified screen-printed carbon electrode (SPCE). A biosensor was fabricated through covalent grafting of a mixed self-assembled monolayer on AuNPs with a specific antibody. To detect JEV and achieve signal amplification, the horseradish peroxidase (HRP)-labeled second antibody was linked to the biosensor through a sandwich immunity reaction. HRP was used to catalyze 4-chloro-1-naphthol oxidation to produce an insoluble precipitate, which introduced a barrier to electron transfer on the electrode. Electrochemical impedance spectroscopy (EIS) was used to monitor the precipitation on the electrode. The electron-transfer resistance $\left(R_{\mathrm{et}}\right)$ of the biosensor was directly correlated with the concentration of JEV in the solution. Under optimal conditions, the method generated a linear response range between 500 and $5 \times 10^{5} \mathrm{pfu} \mathrm{mL}^{-1}$, and the detection limit was $167 \mathrm{pfu} \mathrm{mL}^{-1}$. The biosensor exhibited good selectivity against other viruses.
\end{abstract}

Keywords Impedimetric biosensor, Japanese encephalitis virus, screen-printed carbon electrode, gold nanoparticle, signal amplification

(Received April 20, 2016; Accepted July 11, 2016; Published October 10, 2016)

\section{Introduction}

The Japanese encephalitis virus (JEV) is a member of the genus mosquito-borne flavivirus and closely related to West Nile and Saint Louis encephalitis viruses. ${ }^{1}$ While most JEV infections in humans are asymptomatic, approximately $25 \%$ of all severe clinical cases result in a fatal outcome. Among survivors, $30-50 \%$ present serious neurological, cognitive, or psychiatric sequelae. ${ }^{2}$ To monitor infections and prevent rapid viral spread in these cases, development of methods to detect JEV in suspect cases and potential vectors is necessary.

JEV infections are mainly diagnosed by virus isolation, detection of virus-specific $\operatorname{IgM}$ and $\operatorname{IgG}$ antibodies, and detection of the virus genome by nucleic acid amplification. ${ }^{3,4}$ Further, the hemagglutination inhibition (HI) test and virus neutralization have been employed to detect the presence of anti-JEV antibodies in serum. ${ }^{5}$ However, the $\mathrm{HI}$ test is cumbersome and requires paired serum samples; thus, this approach cannot provide early diagnosis. Virus neutralization requires special laboratory conditions and a high level of technical skill. ${ }^{6}$ Sufficiently sensitive and specific assays based on genomic sequence detection, such as conventional reverse-transcription polymerase chain reaction (RT-PCR), TaqMan-based real-time

† To whom correspondence should be addressed.

E-mail: gaoqiang@snnu.edu.cn (Q. G.); yflei@fmmu.edu.cn (Y. L.)
RT-PCR, SYBR green-based real-time PCR, and reverse loop-mediated isothermal amplification (LAMP), have been developed for the diagnostic detection of JEV genes., ${ }^{4,7}$ However, these methods either require high cost of instruments and technical expertise or present an extremely high risk of contamination. Thus, there is an urgent need to develop a highly sensitive and less expensive detection method for the direct detection of JEV virus in the acute phase of the illness to enable timely clinical treatment and epidemiological investigations.

Electrochemical affinity biosensors designed to detect biomolecules, such as DNA, bacteria, and viruses, have become increasingly useful because of their low cost and good adaptation to miniaturization. Moreover, changes in electrical/ electrochemical properties resulting from biochemical reactions on the surface of the sensor are easily measurable. ${ }^{9-11}$ For the development of the electrochemical affinity biosensor, electrochemical impedance spectroscopy (EIS) is attracting more attention because it is a sensitive technique used to study the interfacial properties of the electrode. ${ }^{12}$ Owing to the complexity of real samples, fouling circumvention and sensitivity must be considered during biosensor preparation. ${ }^{13}$ To design a immunoassay with high sensitivity, signal amplification is a crucial area of interest. ${ }^{14}$ As one of the most popular tracer labels, horseradish peroxidase (HRP) has been extensively used for enzymatic assays. ${ }^{15}$ Amplification through the enzymatic precipitation of a nonconductive insoluble product has also been used for the development of biosensors. ${ }^{16}$ 


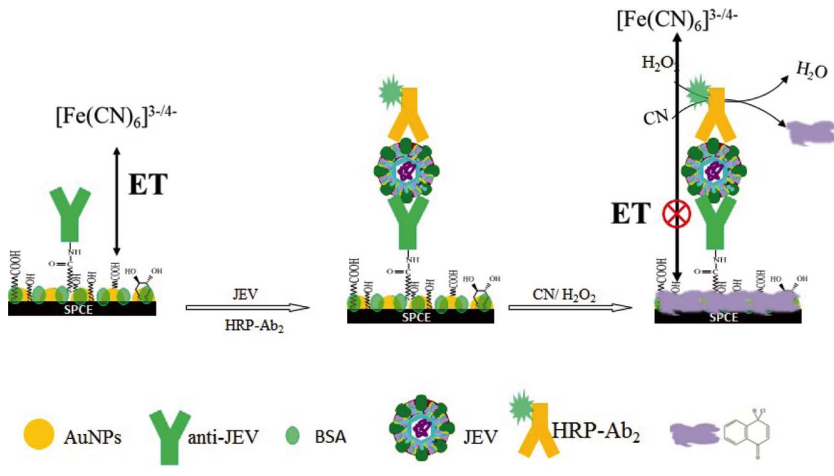

Scheme 1 Preparation of the electrochemical impedance biosensor and detection principle.

In this strategy, an insoluble product is precipitated by an enzyme HRP (biocatalyzed precipitation), forming an insulating layer on an electrode surface, which completely blocks the electron transfer process of a redox probe (such as ferricyanideion). The extent of electrode insulation is monitored by Faradaic impedance spectroscopy and cyclic voltammetry. ${ }^{9}$ However, cost has to be considered for practical application because these kinds of sensors are not renewable. ${ }^{14,17}$ Screenprinted carbon electrodes (SPCEs) simplify the use of electrochemical biosensors, circumvent the need to renew the sensors because of their disposability, and highlight the possibility of carrying out decentralized assays. ${ }^{18}$ However, the immobilization of a biorecognizable element on an SPCE is a challenge. Various methods were employed for the immobilization of biomolecules on SPCE surface, such as physical wrapping, ${ }^{19}$ chemical cross-linking or entrapment, ${ }^{20}$ and covalent immobilization on a functionalized layer on an SPCE. $^{21}$ Gold nanoparticles (AuNPs) are particularly suitable for modification of SPCEs because of their good conductivity, biocompatibility, and high binding affinity to thiol-containing molecules. These NPs can also increase the surface area of the electrode and improve the characterization of an SPCE. ${ }^{22}$ Therefore, AuNP-modified SPECs have been extensively used to prepare the electrochemical biosensor.

In this work, a sensitive electrochemical impedimetric biosensor based on AuNP-modified SPCEs was developed to detect JEV. HRP tag-initiated deposition of an insulating film on the biosensor was used to amplify the impedance signal. As shown in Scheme 1, the AuNP-modified SPCE was functionalized with - $\mathrm{COOH}$ groups by the mixed assembly of 11-mercaptoundecanoic acid (MUA), 6-mercaptohexanol (MCH), and dithiothreitol (DTT) on the electrode surface. An antibody was immobilized by forming an amide bond between the $-\mathrm{NH}_{2}$ moiety of the antibody and the $-\mathrm{COOH}$ group of MUA activated with $N$-hydroxysulfosuccinimide (NHS)/1-ethyl-3-[3dimethylaminopropyl] carbodiimide hydrochloride (EDC) on the AuNPs. To reduce physical adsorption and background impedance, the AuNP surface was backfilled with $\mathrm{MCH}$ and DTT, and the electrode surface was backfilled with bovine serum albumin (BSA). After the JEV in a sample solution was captured by the immobilized antibody on the biosensor, the HRP-labeled secondary antibody was bound to the electrode surface through an immunoreaction, forming an antibody/JEV/ HRP labeled antibody sandwich system. HRP was introduced to the electrode surface for signal amplification. In the presence of 4-chloro-1-naphthol $(\mathrm{CN})$ and $\mathrm{H}_{2} \mathrm{O}_{2}$, HRP induced $\mathrm{H}_{2} \mathrm{O}_{2}$ biocatalytic oxidation of $\mathrm{CN}$ to produce an insoluble product on the electrode. The insulating layer formed by the insoluble product formed a barrier to electron transfer between the redox probe in the electrolyte solution and the electrode. ${ }^{14,17}$ Thus, signal amplification was obtained. The fabrication and performance of the biosensor are also discussed.

\section{Experimental}

\section{Reagents and chemicals}

11-Mercaptoundecanoic acid (MUA), 6-mercaptohexanol (MCH), dithiothreitol(DTT), 1-ethyl-3-[3-dimethylaminopropyl] carbodiimide hydrochloride (EDC), $\mathrm{N}$-hydroxysulfosuccinimide (NHS), 4-chloro-1-naphthol (CN), bovine serum albumin (BSA) and $\mathrm{HAuCl}_{4}$ were purchased from Sigma-Aldrich (Shanghai, China). Dimethyl sulfoxide was obtained from Alfa Aesar (Tianjin, China). Antibodies against JEV $\left(\mathrm{Ab}_{1}\right)$, JEV, HRPlabeled anti-JEV antibody (HPR-Ab $b_{2}$ ), Hantaan virus (HTNV), herpesvirus (HSV), dengue virus (DENV), and adenoviral virus $(\mathrm{AdV})$ were prepared in our laboratory. Other chemicals and reagents used in this work were commercially available and of analytical grade. Water was obtained from a Millipore Milli-Q purification system.

\section{Apparatus}

Electrochemical impedance spectroscopy (EIS) was performed on a Zennium electrochemical workstation (Zahner, Germany). All electrochemical experiments were performed using a conventional three-electrode system, with the SPCE as the working electrode, a platinum foil as the counter electrode, and an $\mathrm{Ag} / \mathrm{AgCl}$ electrode (saturated with $\mathrm{KCl}$ ) as the reference electrode. All potentials in this study were presented in terms of $\mathrm{Ag} / \mathrm{AgCl} /$ saturated $\mathrm{KCl}$ electrode potentials. EIS were performed in the presence of $5 \mathrm{mM}\left[\mathrm{Fe}(\mathrm{CN})_{6}\right]^{3 / 4}$ redox probe (equimolecular mixture in $\mathrm{pH} 7.4,10 \mathrm{mM}$ phosphate-buffered solution (PBS) containing $0.1 \mathrm{M} \mathrm{KNO}_{3}$ ). The direct current (DC) potential was set to $+0.24 \mathrm{~V}$, which is the formal potential of the $\left[\mathrm{Fe}(\mathrm{CN})_{6}\right]^{3 / 4}$ redox probe. The experimental spectra, which are presented as Nyquist plots, were fitted with proper equivalent circuits by using the software supported by the instruments.

Scanning electron microscopy (SEM) was performed using a Quanta 200 (FEI, Philips) apparatus. The acceleration voltage was $20 \mathrm{kV}$. The samples were Au-sputtered prior to the SEM measurements.

\section{Preparation of the AuNP-modified SPCE}

The SPCE used as a substrate electrode was prepared using the method described in the paper. ${ }^{23}$ The procedure for AuNP deposition on the SPCE was adopted from a previous work. ${ }^{24}$ In brief, the SPCE $(1 \times 5 \mathrm{~mm})$ was immersed in $0.5 \mathrm{M} \mathrm{H}_{2} \mathrm{SO}_{4}$ containing $0.5 \mathrm{mM} \mathrm{HAuCl}_{4}$ and then degassed in $\mathrm{N}_{2}$ stream for a minimum of $20 \mathrm{~min}$ prior to electrochemical deposition. A constant potential of $+1.1 \mathrm{~V}$ (versus $\mathrm{Ag} / \mathrm{AgCl}$ ) was applied for $60 \mathrm{~s}$, and then a constant potential of $0 \mathrm{~V}$ (versus $\mathrm{Ag} / \mathrm{AgCl}$ ) was applied for $30 \mathrm{~s}$. Subsequently, the resulting electrode was washed with PBS.

\section{Preparation of the biosensor}

A mixed monolayer of MUA, MCH, and DTT was formed over the AuNPs on the SPCE surface by immersing the AuNPmodified SPCEs in solutions containing MUA $(5 \mu \mathrm{M}), \mathrm{MCH}$ $(0.2 \mathrm{mM})$, and DTT $(0.4 \mathrm{mM})$ for $9 \mathrm{~h}$. Afterward, -COOH on the electrode surface was activated by steeping the electrode in an EDC/NHS aqueous solution (5 mM EDC, $10 \mathrm{mM}$ NHS) for 


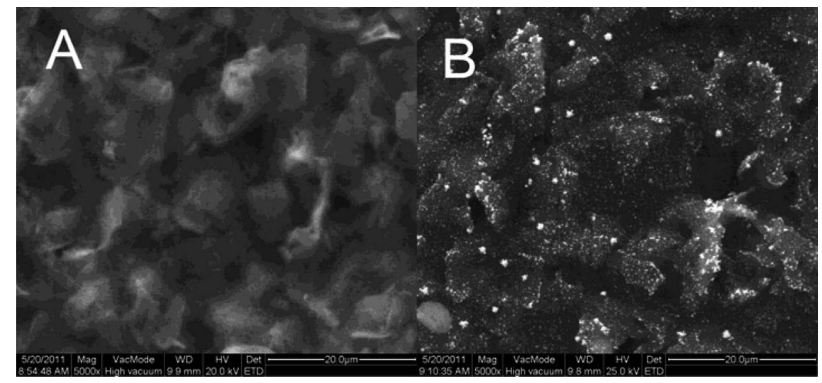

Fig. 1 SEM images of SPCE before (A) and after (B) electrodeposition of AuNPs.

$1 \mathrm{~h}$ at $35^{\circ} \mathrm{C}$. Then, $10 \mu \mathrm{L}$ of $\mathrm{Ab}_{1}(20 \mu \mathrm{g} / \mathrm{mL})$ was dropped on the electrode for $50 \mathrm{~min}$ at $35^{\circ} \mathrm{C}$ under a humidified atmosphere to allow the $-\mathrm{NH}_{2}$ moiety at $\mathrm{Ab}_{1}$ to react with the activated $-\mathrm{COOH}$ group, forming an amide bond. The resultant electrode was immersed in $1 \%$ bovine serum albumin (BSA) solution (diluted with PBS; $10 \mathrm{mM}, \mathrm{pH}$ 7.4) to block active sites on the electrode and reduce the nonspecific adsorption of other antibodies. Non-covalently bound material was removed through washing with PBS. The resulting electrode was a biosensor that could be used for JEV detection.

\section{Measurement of JEV}

The JEV sample solution $(10 \mu \mathrm{L})$ was spotted on the biosensor for $50 \mathrm{~min}$ at $35^{\circ} \mathrm{C}$. Then, the HRP-Ab $\mathrm{b}_{2}$ solution $(10 \mu \mathrm{L})$ was dropped onto the biosensor surface to form a sandwich sensing system; this interaction was maintained at $35^{\circ} \mathrm{C}$ for $50 \mathrm{~min}$. The biosensor was washed with PBS buffer (10 mM PBS, $\mathrm{pH} 7.4$, containing $0.5 \%$ Tween 20) to remove the physical adsorption after each step.

The above biosensor was incubated in the substrate solution, which consisted of $2 \mathrm{mM} \mathrm{CN}$ and $15 \mathrm{mM} \mathrm{H}_{2} \mathrm{O}_{2}$, for $60 \mathrm{~min}$ After incubation in the substrate solution, the electrode was rinsed and subjected to electrochemical measurements. In the control experiments, the electrodes were incubated in the absence of JEV. The electrode was thoroughly rinsed with PBS buffer after each step.

\section{Results and Discussion}

\section{SEM of the AuNP-modified SPCE}

The morphology of the AuNPs on the SPCEs was characterized by SEM. Figure 1 displays the typical SEM images of a bare SPCE and an SPCE after Au electrodeposition. A rough and jagged structure with randomly distributed carbon particles was observed for the bare SPCEs (Fig. 1A). After the electrodeposition of $\mathrm{Au}$ on the SPCEs, the surfaces of the electrodes were mostly covered with homogeneous particles (Fig. 1B). The results indicate that the AuNPs were electrodeposited on the SPCE surface.

\section{EIS characterization of the biosensor}

Here, EIS was used to investigate the characteristics of the biosensor in each assembly step, and results are shown in Fig. 2. The Randles equivalent circuit (Fig. 2 and inset) was selected to fit the impedance data. This circuit consists of the electrolyte solution resistance $\left(R_{\mathrm{s}}\right)$, diffusion-related Warburg impedance $\left(Z_{\mathrm{w}}\right)$, interfacial capacitance $\left(C_{\mathrm{dl}}\right)$, and the electron-transfer resistance $\left(R_{\mathrm{et}}\right)$, corresponding to the diameter of the semicircle
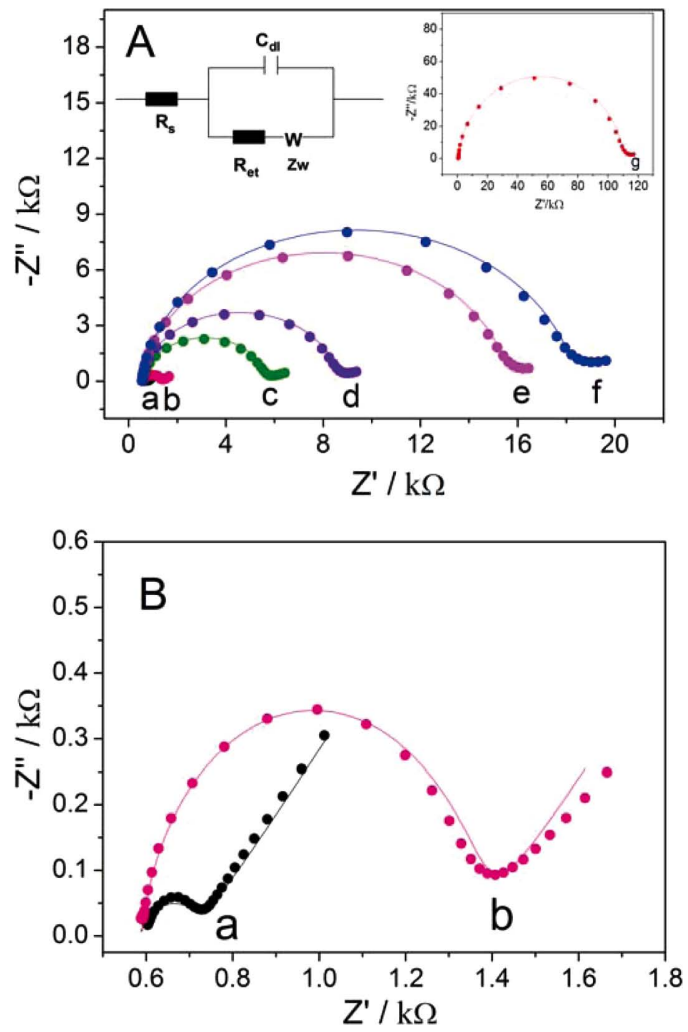

Fig. 2 Nyquist plots of the electrochemical impedance spectra of the (a) AuNP-modified SPCE electrode, (b) MUA-MCH-DTT/AuNPmodified SPCE, (c) Ab 1 /MUA-MCH-DTT/AuNP-modified SPCE, (d) BSA/Ab $/$ /MUA-MCH-DTT/AuNP-modified SPCE, (e) JEV/BSA/ $\mathrm{Ab}_{1}$ /MUA-MCH-DTT/AuNP-modified SPCE, (f) $\mathrm{HRP}-\mathrm{Ab}_{2} / \mathrm{JEV} /$ BSA/Ab 1 /MUA-MCH-DTT/AuNP-modified SPCE, and (g) the biosensor obtained after incubation in an substrate solution containing $\mathrm{CN}$ and $\mathrm{H}_{2} \mathrm{O}_{2}$. (B) Enlarged view of curves $\mathrm{a}$ and $b$. Concentration of $\mathrm{JEV}, 5 \times 10^{4}$ pfu $\mathrm{mL}^{-1}$.

at the higher frequency parts of the Nyquist plot. Given that $R_{\mathrm{et}}$ is the most direct and sensitive parameter responding to changes on the electrode interface, it was used to denote signals. ${ }^{25}$ Figure 2 reveals that the experimental impedance data (dot) are consistent with the fitted data (line). The Nyquist plot of the AuNP-modified electrode presented a virtually straight line (curve a), indicating that the electrochemical process is controlled by a diffusion-limiting process. After the mixed selfassembly of MUA, MCH, and DTT onto the electrode (curve b), a semicircle appeared at higher frequencies corresponding to a $R_{\text {et }}$ of $774 \Omega$. This result demonstrates that a self-assembled monolayer was formed on the electrode surface.

After immobilization of $\mathrm{Ab}_{1}$, an increase in $R_{\text {et }}$ to $5007 \Omega$ was observed (Fig. 2c). This result indicates that $A b_{1}$ was immobilized onto the electrode because the antibody forms an insulating layer on the AuNP-modified SPCE surface, leading to increased $R_{\mathrm{et}}{ }^{26}$ Subsequently, BSA adsorption led to an increase in $R_{\text {et }}$ from 5007 to $7977 \Omega$ (Fig. 2 d).

For JEV detection, the sample solution was dropped onto the biosensor surface, causing an increase in $R_{\mathrm{et}}$ to $14800 \Omega$ (Fig. 2e), indicating that JEV was captured by the immobilized antibody on the biosensor surface via a specific immune reaction. Afterward, HRP-Ab $\mathrm{b}_{2}$ was linked to the biosensor surface, and an increase in $R_{\mathrm{et}}$ to $17460 \Omega$ was obtained (Fig. 2f). Finally, the biosensor was incubated in solutions containing $\mathrm{CN}$ and $\mathrm{H}_{2} \mathrm{O}_{2}$, and this treatment resulted in a 

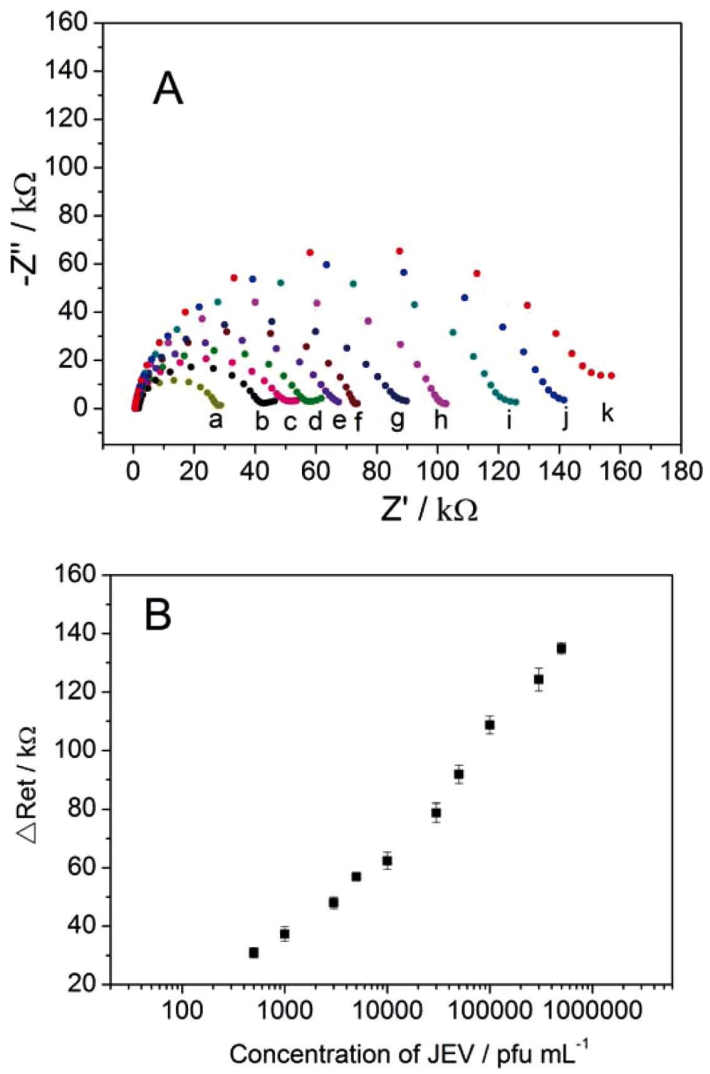

Fig. 3 EIS response curves of different concentrations of JEV (A) and $R_{\mathrm{et}}$ as a function of JEV concentration (B). Concentration of JEV (a) blank, (b) $5 \times 10^{2}$, (c) $1 \times 10^{3}$, (d) $3 \times 10^{3}$, (e) $5 \times 10^{3}$, (f) $1 \times 10^{4}$, (g) $3 \times 10^{4}$, (h) $5 \times 10^{4}$, (i) $1 \times 10^{5}$, (j) $3 \times 10^{5}$, (k) $5 \times 10^{5} \mathrm{pfu} \mathrm{mL}^{-1}$.

significant increase in $R_{\text {et }}$ from 13940 to $109200 \Omega$, as shown in Fig. $2 \mathrm{~g}$. The increase in $R_{\mathrm{et}}$ is due to the precipitation of the insoluble film and the formation of an insulating layer on the electrode. The insoluble precipitate restrained the electron transfer of $\left[\mathrm{Fe}(\mathrm{CN})_{6}\right]^{3 / 4-}$ on the sensor surface. This result indicates that cascading signal amplification was achieved.

\section{EIS detection}

The concentration-dependent behavior of impedance signals when the target JEV was bound to the biosensor was investigated to evaluate the sensitivity of the biosensor for detecting JEV. Under optimal conditions, the signal responses, ranging from $500 \mathrm{pfu} \mathrm{mL} \mathrm{m}^{-1}$ to $5 \times 10^{5} \mathrm{pfu} \mathrm{mL}^{-1}$ (Fig. 3), were linearly related to target the JEV concentration. The regression equation was $\Delta R_{\text {et }}(\mathrm{k} \Omega)=35 \log c\left(\right.$ pfu $\left.\mathrm{mL}^{-1}\right)-72$ (where $c$ is the concentration of the target JEV), and a correlation coefficient of 0.989 was obtained. The detection limit was $167 \mathrm{pfu} \mathrm{mL}^{-1}(S / N=3)$. The detection limit of the gold-coated magnetic beads-based electrochemical immunoassay for JEV was $2.0 \times 10^{3} \mathrm{pfu} \mathrm{mL}^{-1}$, 13 which was 2 orders of magnitude higher than that of immunochromatographic strip and similar to that obtained from RT-PCR. ${ }^{27}$ The sensitivity of reverse transcription loopmediated isothermal amplification assay was similar to real-time RT-PCR, but was 10-fold higher than conventional RT-PCR. ${ }^{28}$ So, the detection limit of the most sensitive assay for JEV is $2.0 \times 10^{2} \mathrm{pfu} \mathrm{mL}^{-1}$. For the early diagnosis of JEV, more sensitive assay will be needed. Here, we developed the immunosensor whose lower detection limit of JEV is 167 pfu $\mathrm{mL}^{-1}$.

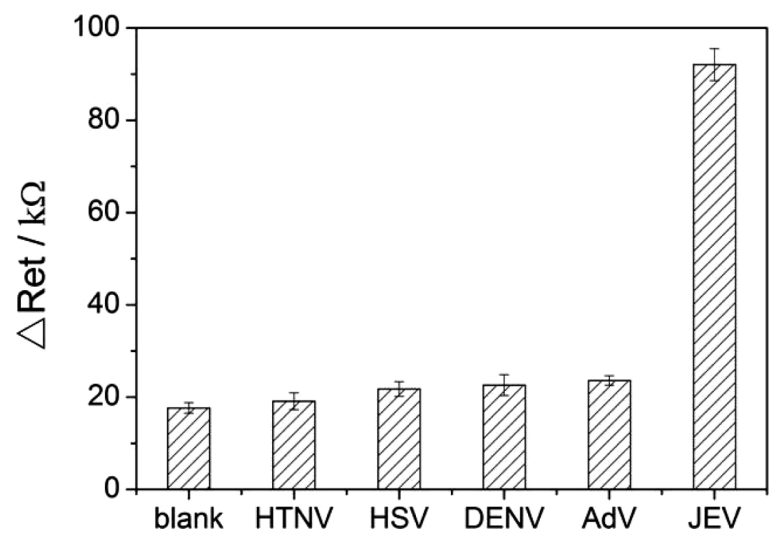

Fig. 4 Selectivity of the biosensor. Each virus sample concentration is $5 \times 10^{4} \mathrm{pfu} \mathrm{mL}^{-1}$. Concentration of JEV, $3 \times 10^{4} \mathrm{pfu} \mathrm{mL}^{-1}$.

We investigated the reproducibility of the developed biosensor and estimated this parameter as the relative standard deviation of four measurements with different biosensors at different concentration levels. At a JEV concentration of $3 \times 10^{4}$ pfu $\mathrm{mL}^{-1}$, the relative standard deviation of six detections with six different sensors was $7.5 \%$. These results demonstrate that measurements using the JEV immunosensor are highly reproducible.

\section{Selectivity}

Selectivity is an important feature to consider when developing biosensors. To evaluate the selectivity of the developed sensor, the impedimetric responses of the biosensor to certain viruses, such as HTNV, HSV, DENV, and AdV, were investigated. As shown in Fig. 4, no significant interference was found from the non-target virus, thereby suggesting the feasibility of specific detection of JEV by using the biosensor.

\section{Conclusions}

A specific and sensitive impedimetric immune-biosensor for JEV detection was developed based on enzymatic precipitation for signal amplification on a disposable gold nanoparticlemodified screen-printed carbon electrode. Owing to HRP mediated precipitation of the insoluble product, an insulating film amplified impedance signals and lowered the JEV detection limit of the biosensor to $167 \mathrm{pfu} \mathrm{mL}^{-1}$. The biosensor presents enhanced responses with increasing JEV concentrations within

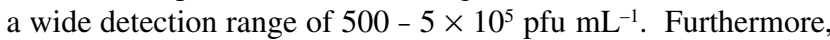
the use of an AuNP-modified SPCE as a base electrode reduced the cost of the developed device.

\section{Acknowledgements}

The authors gratefully acknowledge the financial support from the National Nature Science Foundation of China (No. 21175089), Program for Innovative Research Team in Shaanxi Province (No. 2014KCT-28), and the Natural Science Basic Research Plan in Shaanxi Province of China (Nos. 2013SZS08-Z01 and 2013SZS08-P01). 


\section{References}

1. T. P. Endy and A. Nisalak, Curr. Top. Microbiol., 2002, 267, 11.

2. G. Watt and K. Jongsakul, Am. J. Trop. Med. Hyg., 2003, 68, 704 .

3. Y. Li, L. Hou, J. Ye, X. Liu, H. Dan, M. Jin, H. Chen, and S. Cao, J. Virol. Methods, 2010, 168, 51.

4. S. H. Huang, T. C. Yang, M. H. Tsai, I. S. Tsai, H. C. Lu, P. H. Chuang, L. Wan, Y. J. Lin, C. H. Lai, and C. W. Lin, Nanotechnology, 2008, 19, 4050101.

5. J. Hirota, H. Nishi, H. Matsuda, H. Tsunemitsu, and S. Shimiz, J. Vet. Med. Sci., 2010, 72, 369.

6. B. R. Gulati, H. Singha, B. K. Singh, N. Virmani, S. K. Khurana, and R. K. Singh, J. Vet. Sci., 2011, 12, 341.

7. S. C. Barros, F. Ramos, L. Z. Zéb, M. J. Alvesb, T. Fagulhaa, M. Duartea, Ma. Henriquesa, T. Luísa, and M. Fevereiroa, J. Virol. Methods, 2013, 193, 554.

8. M. M. Parida1, S. R. Santhosh1, P. K. Dash, N. K. Tripathi, P. Saxena, S. Ambuj, A. K. Sahni, P. V. Lakshmana Rao, and K. Morita, J. Clin. Microbiol., 2006, 44, 4172.

9. L. Hou, Z. Q. Gao, M. D. Xu, X. Cao, X. P. Wu, G. N. Chen, and D. P. Tang, Biosens. Bioelectron., 2014, 54, 365.

10. J. P. Metters, R. O. Kadara, and C. E. Banks, Analyst, 2011, 136, 1067.

11. T. Q. Huy, N. T. H. Hanh, N. T. Thuy, P. V. Chung, P. T. Nga, and M. A. Tuan, Talanta, 2011, 86, 271.

12. L. Ianeselli, G. Grenci, C. Callegaric, M. Tormenb, and L. Casalis, Biosens. Bioelectron., 2014, 55, 1.

13. F. Li, L. Mei, Y. M. Li, K. H. Zhao, H. C. Chen, P. Wu, Y. G. $\mathrm{Hu}$, and S. B. Cao, Biosens. Bioelectron., 2011, 26 , 4253.
14. L. Hou, Y. Tang, M. D. Xu, Z. Q. Gao, and D. P. Tang, Anal. Chem., 2014, 86, 8352.

15. R. Akter, M. A. Rahman, and C. K. Rhee, Anal. Chem., 2012, 84, 6407.

16. L. Hou, X. P. Wu, G. N. Chen, H. H. Yang, M. H. Lu, and D. P. Tang, Biosens. Bioelectron., 2015, 68, 487.

17. F. Yang, J. Han, Y. Zhuo, Z. H. Yang, Y. Q. Chai, and R. Yuan, Biosens. Bioelectron., 2014, 55, 360.

18. M. Tudorache and C. Bala, Anal. Bioanal. Chem., 2007, 388, 565.

19. G. Marrazza, I. Chianella, and M. Mascini, Anal. Chim Acta, 1999, 387, 297.

20. S. Jampasa, W. Wonsawat, N. Rodthongkum, W. Siangproh, P. Yanatatsaneejit, T. Vilaivan, and O. Chailapakul, Biosens. Bioelectron., 2014, 54, 428

21. Y. H. Zhang, X. H. Geng, J. J. Ai, Q. Gao, H. L Qi, and C. X. Zhang, Sens. Actuators, B, 2015, 221, 1535.

22. J. Liu, X. Q. Yuan, Q. Gao, H. L. Qi, and C. X. Zhang, Sens. Actuators, B, 2012, 162, 384.

23. Q. Gao, Y. Guo, W. Y. Zhang, H. L. Qi, and C. X. Zhang, Sens. Actuators, B, 2011, 153, 219.

24. J. Liu, X. Q. Yuan, Q. Gao, H. L. Qi, and C. X. Zhang, Sens. Actuators, B, 2012, 162, 384.

25. R. Jurczakowski and P. Połczyński, J. Phys. Chem. C, 2014, $118,7980$.

26. B. Rezaei, N. Majidi, H. Rahmani, and T. Khayamian, Biosens. Bioelectron., 2011, 26, 2130.

27. H. Liu, Z. J. Liu, J. Jing, J. Q. Ren, Y. Y. Liu, H. H. Guo, M. Fan, H. J. Lu, and N. Y. Jin, Vector. Borne. Zoonotic Dis., 2012, 12, 1042.

28. J. Deng, J. J. Pei, H. C. Gou, Z. D. Ye, and C. C. Liu, and J. D. Chen, J. Virol. Methods, 2015, 213, 98 